\title{
Importância do Indice Anatômico de Gravidade do Trauma no manejo das fraturas toracolombares do tipo explosão
}

\author{
The importance of Injury Severity Score (ISS) in the management of \\ thoracolumbar burst fracture
}

Rodrigo ReZende ${ }^{1}$; Osmar AvanzI ${ }^{2}$

RES U M O

\begin{abstract}
Objetivo: Avaliar o valor da Injury Severity Score (ISS) na determinação da gravidade das fraturas vertebrais do tipo explosão considerando a amplitude do trauma e a escolha do melhor tratamento para diminuir a morbimortalidade do acidente. Métodos: 190 pacientes foram incluídos, nos quais avaliaram-se a idade, o mecanismo do trauma, o tipo de fratura na coluna toracolombar, avaliação neurológica, onde se localizavam as lesões frente a ISS e a divisão em categorias. Resultados: A média do ISS foi de 14,4 sendo o menor valor de 4 e o maior de 43, e 126 pacientes apresentavam ISS menor do que 15 (63,3\%), 34 entre 15 e 24 (17,9\%), 25 entre 25 e $34(13,2 \%)$ e cinco com ISS maior que 35 (2,6\%). O valor do ISS foi inversamente proporcional a idade. As fraturas ocorridas na transição toracolombar apresentavam média do ISS de 13,1, sendo menor do que a média do ISS na região torácica de 17,6 e lombar com 16. Dos 102 pacientes tratados sem procedimento cirúrgico, a média do ISS foi de 12,3, enquanto que nos 88 tratados cirurgicamente ela foi de17,6. A mortalidade apresentou-se diretamente proporcional ao valor do ISS. A média dos que evoluíram para óbito foi de 28,5, não ocorrendo óbito entre os classificados com trauma leve. A mortalidade foi de 4,2 \%. Conclusão: O valor de 14,4 e a análise das categorias do ISS sinalizam que trauma de pequena gravidade pode causar fratura da coluna vertebral torácica ou lombar do tipo explosão. O valor do ISS não demonstrou correlação com o nível da fratura, estando diretamente proporcional com o tempo de internação, com o tratamento cirúrgico e a taxa de mortalidade, apresentando-se inversamente proporcional com a idade dos pacientes.
\end{abstract}

Descritores: Vértebras lombares. Coluna vertebral. Vértebras torácicas. Traumatismos da coluna vertebral. Traumatismo múltiplo.

\section{INTRODUÇÃO}

$\mathrm{O}$ aumento crescente dos acidentes industriais e auto mobilísticos faz do trauma a principal causa de invalidez e de óbitos em crianças e adulto jovens, levando a elevados gastos públicos e alto índice de morbidade e mortalidade ${ }^{1-5}$.

A importância do Injury Severity Score (ISS) ${ }^{5}$ nos dias atuais consiste em quantificar a gravidade do trauma, estimar a mortalidade nestes pacientes e auxiliar o médico socorrista no momento de tomada de decisão sobre a meIhor forma de tratamento. Além disso, auxilia no planejamento de remoção de pacientes politraumatizados para centros de tratamento terciário, especializados nestes traumatismos ${ }^{5}$.

Os trabalhos na literatura demonstram a importância da análise do ISS no trauma ortopédico, particularmente na definição da gravidade das lesões ocorridas no politraumatizado, no tipo de tratamento indicado na ur- gência e na sua relação com a morbi-mortalidade ${ }^{6,7}$, porém poucos são os encontrados que estudaram a gravidade do trauma em pacientes com fraturas vertebrais, em especial nos portadores de fratura tipo explosão.

Nestas fraturas, não existe consenso na literatura quanto à melhor forma de tratamento. Diversos fatores, como perda da altura da parede anterior da vértebra fraturada, porcentagem de fragmento ósseo no canal medular, grau de cifose e quadro neurológico interferem no momento da tomada de decisão. Fatores como localização da lesão, grau de estabilidade, fratura associada da lâmina e do processo transverso também interferem na escolha da forma do tratamento a ser realizado ${ }^{8,9}$. A diversidade desses fatores explica a grande controvérsia e discussão existente no tratamento das fraturas do tipo explosão, pois não existe até o momento um tratamento universalmente aceito.

Outro dissenso na literatura é em relação ao tipo de abordagem cirúrgica nesses casos. Muitos autores tem

Trabalho realizado no Departamento de Ortopedia e Traumatologiada Faculdade de Ciências Médicas da Santa Casa de Misericórdia de São Paulo (SCMSP), São Paulo, SP, Brasil.

1. Mestre do Curso de Pós-Graduação.Área de Concentração em Ortopedia e Traumatologia. Do Departmento de Ortopedia e Traumatologia da Faculdade de Ciências Médicas da Santa Casa de Misericórdia de São Paulo (SCMSP), SP, Brasil; 2. Professor Adjunto do Departamento de Ortopedia e Traumatologia da Faculdade de Ciências Médicas da Santa Casa de Misericórdia de São Paulo (SCMSP), SP, Brasil. 
preconizado a abordagem posterior principalmente nos pacientes que não apresentam déficit neurológico ${ }^{10-12}$. Neles a abordagem por via anterior e a descompressão direta do canal medular pode ser realizada ${ }^{13}$.

Sabe-se que muitas complicações poderiam ser evitadas se a análise da gravidade do trauma fosse melhor conhecida e aplicada pelo médico socorrista e pela equipe de cirurgiões de coluna vertebral. Por isso, seria importante conhecer qual o valor do ISS nos portadores de fratura por explosão da coluna toracolombar e questiona-se nestas fraturas se o ISS apresenta valor elevado como nas fraturas luxações da coluna vertebral. Mais precisamente será que no tipo explosão o cálculo e a análise dos valores do ISS traria alguma contribuição em termos de tratamento definitivo destas lesões?

Como há ausência de critérios na literatura para definir quais as fraturas são mais graves, o estudo da associação do ISS com os valores da radiologia simples e TAC poderia colaborar na avaliação da gravidade do trauma vertebral.

A literatura mostra que os pacientes politraumatizados com ISS superior a 15 apresentam maior gravidade e consequentemente maior morbimortalidade; porém não são especificados qual o tipo de fratura da coluna vertebral e quais os critérios adotados para definir a gravidade do trauma nestas fraturas ${ }^{13-15}$. Assim, o ISS de 15 pode não ser o mesmo quando se avaliam diferentes tipos de fraturas da coluna vertebral ${ }^{13}$.

Em função desta controvérsia, este estudo tem por objetivo avaliar o valor da Injury Severity Score (ISS) na determinação da gravidade das fraturas vertebrais do tipo explosão considerando a amplitude do trauma e a escolha do melhor tratamento para diminuir a morbimortalidade do acidente.

\section{MÉTODOS}

No período compreendido entre os anos de 1990 e 2004, foram atendidos 555 pacientes com fratura da coluna vertebral e, destes, 190 eram do tipo explosão classificada pelos critérios de Denis ${ }^{1}$. Conforme estes critérios, nas radiografias observa-se a abertura dos pedículos na incidência de frente (Figura 1) e o colapso do corpo vertebral na de perfil. Na tomografia axial computadorizada, definese o diagnóstico com a vizibilização do fragmento ósseo para o interior do canal vertebral no local da fratura explosiva da vértebra (Figura2).

Cento e trinta e seis pacientes eram do sexo masculino (71,6\%); a média da idade foi de 39,2 anos; o mecanismo de trauma mais frequente foi a queda de altura $(74,7 \%)$, seguida por acidente automobilístico com 13,7\%. A fratura na coluna toracolombar correspondeu à maioria dos pacientes $(59,5 \%)$, seguida pelas fraturas lombares em $36,8 \%$ e 146 pacientes encontravam-se com exame neurológico normal $(76,8 \%)$. Dos 190 pacientes, 102 foram tratados con- servadoramente $(53,7 \%)$ e em 88 pacientes optou-se pelo tratamento cirúrgico $(46,3 \%)$.

O método adotado para a classificação da gravidade do trauma foi o ISS que é derivado do AIS ${ }^{16,17}$. De acordo com o ISS, para o cálculo da gravidade do trauma, deve-se utilizar no máximo até três regiões diferentes do corpo humano com as lesões mais graves. O cálculo da perda da altura da parede anterior da vértebra acometida, a avaliação neurológica e a subdivisão da localização das fraturas torácicas e lombares seguiram propostas publicadas na atualidade ${ }^{14,18,19}$.

Dividiram-se os resultados do ISS em quatro categorias: ISS menor de 15 para trauma de menor gravidade; ISS entre 15 e 24 caracterizando moderada gravidade;

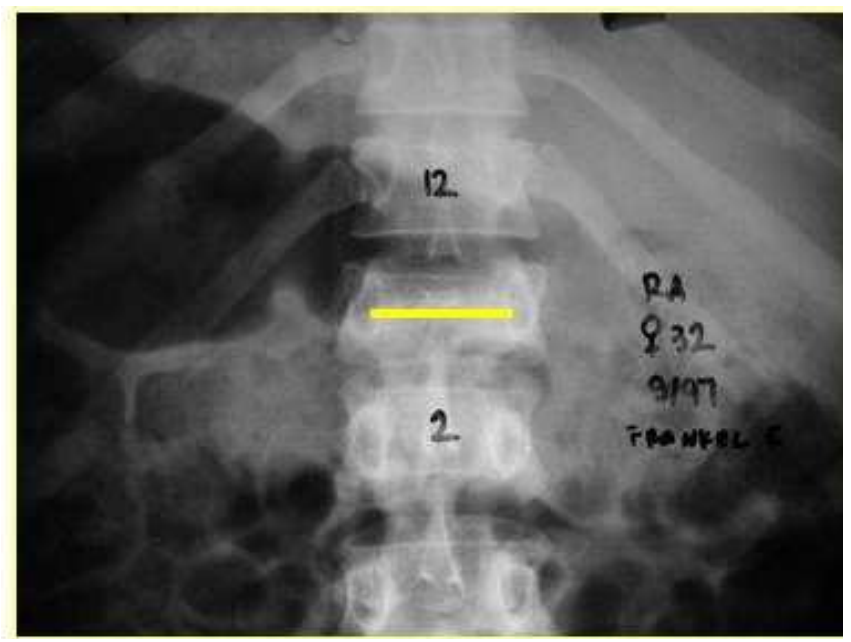

Figura 1 - Imagem radiográfica na incidência ântero-posterior da coluna vertebral evidenciando a abertura da distância entre os pedículos vertebrais característico da fratura do tipo explosão.

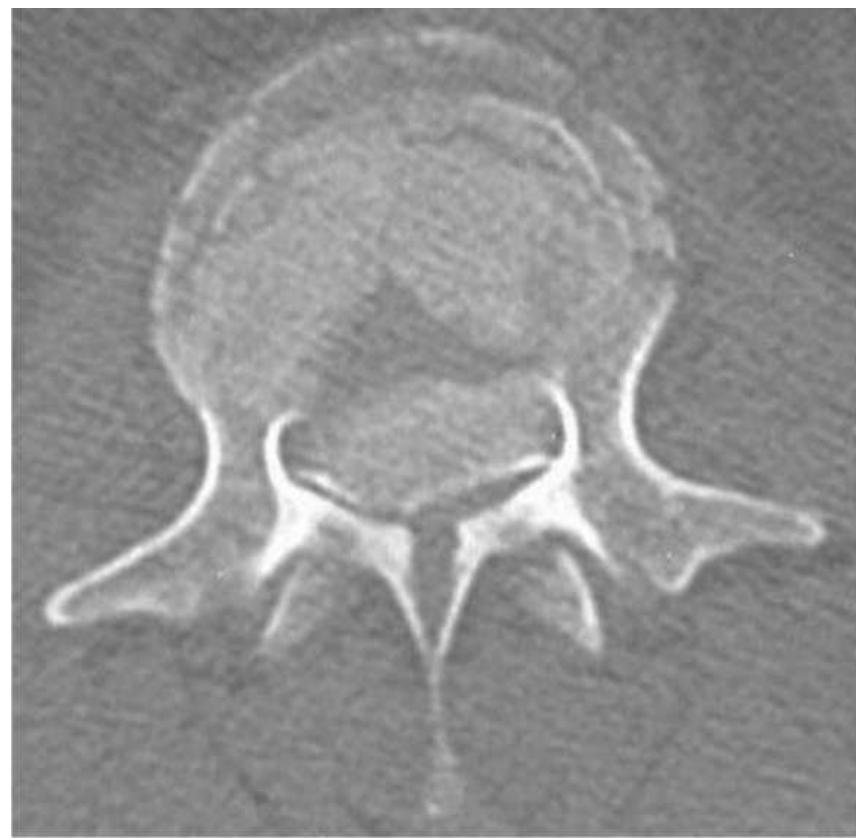

Figura 2 - Imagem de tomografia axial computadorizada evidenciando a retropulsão de fragmentos em direção ao canal vertebral característicos da fratura do tipo explosão. 
ISS entre 25 e 34 como grave; ISS maior de 35 para trauma gravíssimo.

\section{RESULTADOS}

A média do ISS foi de 14,4 sendo o menor valor de 4 e o maior de 43 . Cento e vinte e seis pacientes apresentavam ISS menor do que 15 (63,3\%), 34 entre 15 e 24 $(17,9 \%), 25$ entre 25 e $34(13,2 \%)$ e cinco pacientes com ISS maior que $35(2,6 \%)(p<0,001)$.

O valor do ISS foi inversamente proporcional a idade, ou seja, quanto maior ISS mais jovens eram os pacientes $\left(p^{\star}=0,011\right)$. Aqueles com idade entre 15 e 44 anos apresentaram média de ISS de 15,2, enquanto os com idade entre 45 e 64 anos, média de $13\left(p^{*}=0,011\right)$ (Figura 3).

O tempo de internação médio foi de 20,7 dias, e observou-se que quanto maior a gravidade do trauma maior foi tempo de internação exceto nos pacientes com ISS acima de $35\left(p^{*}<0,001\right)$ (Figura 4$)$.

Quanto ao nível de acometimento, as fraturas tipo explosão ocorridas na transição toracolombar (T11-L1) apresentavam média do ISS de 13,1, sendo menor do que a média do ISS na região torácica de 17,6 e lombar com 16 $(p=0,37)$.

Dos 102 pacientes tratados sem procedimento cirúrgico, a média do ISS foi de 12,3, enquanto que nos 88 tratados cirurgicamente o valor da média do ISS foi de 17,6 $(p<0,001)$ (Tabela 1).

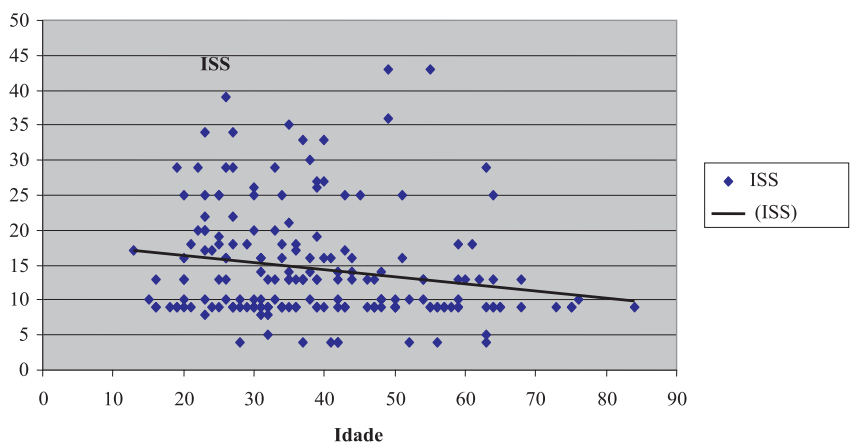

Figura 3 - Relação entre ISS e idade. Fonte: SAME-ISCSP.

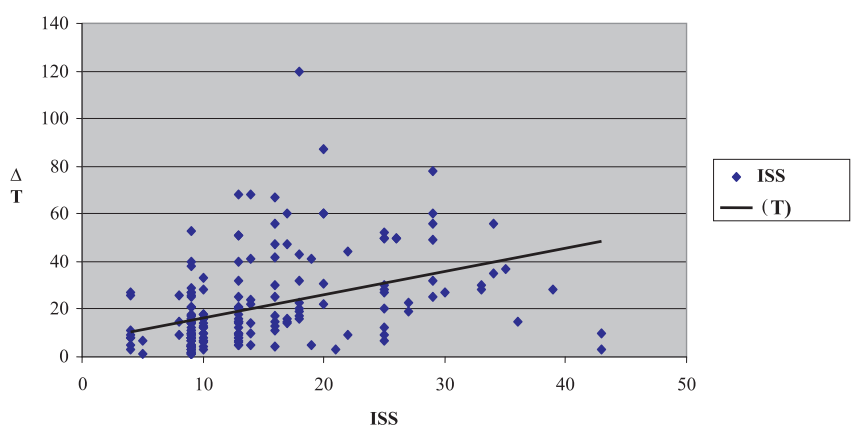

Figura 4 - $\quad$ Relação entre ISS e tempo de internação. Fonte: SAME-ISCSP.
A mortalidade dos pacientes apresentou-se diretamente proporcional ao valor do ISS. A média dos que evoluíram para óbito foi de 28,5, não ocorrendo óbito entre os classificados com trauma leve (ISS $<15)(p=0,007)$.

Oito pacientes morreram, ou seja, a taxa de mortalidade foi de $4,2 \%$. Destes, três apresentavam-se com ISS entre 16 e 24 , três na categoria entre 25 e 34 e nos outros dois o valor do ISS estava entre 35 a 50 (Tabela 2).

\section{DISCUSSÃO}

Com a criação do ISS começa a se definir numericamente a gravidade do trauma dos pacientes vítimas de acidentes automobilísticos ${ }^{5,13}$ A partir desta época o ISS passa a ser aplicado por várias escolas médicas, se tornando o mais reconhecido e difundido método de avaliação da gravidade do trauma ${ }^{13}$.

As fraturas da coluna torácica ou lombar normalmente são causadas por traumas de alta energia e comumente estão associadas a outras lesões. Existe grande preocupação em classificar o tipo, a localização e a forma de tratamento das fraturas vertebrais, porém pouco valor é dado para as lesões associadas a estas fraturas no momento do atendimento e tratamento do politraumatizado.

Os poucos estudos encontrados sobre o valor do ISS nas fraturas da coluna vertebral são muitos divergentes. Vários autores ao analisarem a gravidade do trauma nos diversos tipos de fraturas vertebrais encontraram média do ISS de 21,3, de 31,7 e de1517-19. Já foi encontrado, através de regressão logística, que valores do ISS abaixo e acima de 15 representam a menor e maior gravidade de trauma respectivamente ${ }^{20}$.

Neste estudo de 190 pacientes com fratura exclusivamente do tipo explosão, a média do ISS encontra-

Tabela 1 - Relação entre categorias do ISS e a forma de tratamento.

\begin{tabular}{lrlrlr}
\hline ISS & TT $^{\circ}$ Não Cirúrgico & \multicolumn{3}{c}{ TT $^{\circ}$ Cirúrgico } & \multicolumn{1}{c}{ N } \\
\hline$<15$ & 85 & $(67,5 \%)$ & 41 & $(32,5 \%)$ & $126(100 \%)$ \\
$15-24$ & 6 & $(17,6 \%)$ & 28 & $(82,4 \%)$ & $34(100 \%)$ \\
$25-34$ & 7 & $(28 \%)$ & 18 & $(72 \%)$ & $25(100 \%)$ \\
$35-75$ & 4 & $(80 \%)$ & 1 & $(20 \%)$ & $5(100 \%)$ \\
\hline
\end{tabular}

Tabela 2 - Relação entre categorias do ISS e taxa de mortalidade.

\begin{tabular}{lcrc}
\hline ISS & Obito & $\mathbf{N}$ & \% Obitos \\
\hline$<15$ & - & 126 & $0 \%$ \\
$15-24$ & 3 & 34 & $8,8 \%$ \\
$25-34$ & 3 & 25 & $12 \%$ \\
$35-75$ & 2 & 5 & $40 \%$ \\
\hline
\end{tabular}


da foi de 14,4, sugerindo este valor que a intensidade do trauma para causar explosão na região torácica ou lombar não é alta, se comparada ao mencionado na literatura quando avaliam vários tipos de fraturas vertebrais, que não só as por explosão. Sendo assim o cálculo do ISS representa um passo inicial para a definição da fratura vertebral. Do ponto de vista prático, ISS maior de 14,4 indica alta probabilidade de fratura do tipo explosão, mesmo quando visualizada no estudo radiológico inicial fraturas que possam significar apenas um encunhamento vertebral. Assim, o cálculo do ISS pode guiar investigação mais detalhada de fraturas evidenciadas na radiografia simples indicando a busca de mais elementos para orientar o tratamento.

A literatura sugere que os valores do ISS possam a ser melhor analisados por meio de 4 categorias $^{15,21}$. Os autores deste trabalho concordam com esta forma de análise, porque permite caracterizar se determinado trauma é mais leve ou mais grave, não ficando exclusivamente caracterizado por um número específico do ISS.

Neste estudo, quando distribuiu-se o ISS em categorias, observou-se que $63,3 \%$ dos pacientes encontravamse com ISS abaixo de 15 demonstrando que a maioria das fraturas do tipo explosão foram causadas por trauma de leve intensidade. Apenas 2,6\% sofreram trauma de gravíssima intensidade, ou seja, enquadrados na faixa acima de 35.

Quando aplicou-se a faixa etária destes pacientes nas categorias do ISS, observou-se que nos acima de 65 anos as fraturas do tipo explosão ocorriam por traumas considerados leves (ISS < 15) enquanto que nos com idade entre 15 e 44 anos a gravidade do trauma necessária era maior (ISS >15). Portanto, para haver uma fratura por explosão em pacientes jovens, há necessidade de trauma de maior gravidade, se comparada com os mais idosos. Este fato sugere que as fraturas por explosão em pacientes jovens apresentam comportamento mais grave, servindo como alerta para os médicos socorristas, que nos pacientes jovens com ISS elevado é imperativo avaliação clínica mais detalhada associada a exames de imagem complementares em hospitais terciários.
Quanto à forma de tratamento, estudo ${ }^{16}$ analisando todos os tipos de fraturas vertebrais observou correlação estatisticamente significante entre o tratamento conservador e cirúrgico destas fraturas e os valores do ISS. Nele, o valor médio do ISS foi de 15,3 e 21,5 respectivamente. No estudo aqui apresentado, observou-se que dos 102 pacientes que não foram tratados cirurgicamente $83,3 \%$ apresentavam ISS menor que 15, fato este também encontrado em $20 \%$ dos pacientes com ISS maior que 35. Não houve tempo suficiente para a realização do tratamento definitivo nos pacientes com trauma gravíssimo, sugerindo a importância de se realizar o tratamento de emergência nestes casos. Observou-se que o comportamento da forma do tratamento foi diretamente proporcional ao valor do ISS (pacientes com ISS acima de 15 foram preferencialmente tratados cirurgicamente), não existindo nenhum achado na literatura que correlacione valor do ISS com tratamento cirúrgico.

Além de servir de alerta para o socorrista de risco de maior morbimortalidade nos portadores de fratura toracolombar por explosão com ISS>15, a importância de se determinar o valor do ISS nas fraturas desse tipo é auxiliar a indicação de tratamento conservador ou cirúrgico. Este trabalho sugere que o ISS pode auxiliar na escolha do tratamento operatório nos jovens politraumatizados com ISS maior do que 15 que apresentem fratura vertebral do tipo explosão na transição toracolombar e com medidas mostrando estenose do canal, encunhamento, cifose em valores limites ou naqueles inconscientes após o trauma.

Com base nos achados deste estudo, sugere-se o uso do ISS na rotina do atendimento de pacientes com possibilidade de trauma vertebral por explosão, para averiguar de forma objetiva a gravidade do trauma e uniformizar os dados. O valor de 14,4 e a análise das categorias do ISS sinalizam que um trauma de pequena gravidade pode causar fratura da coluna vertebral torácica ou lombar do tipo explosão. O valor do ISS não demonstrou correlação com o nível da fratura, estando diretamente proporcional com o tempo de internação, com o tratamento cirúrgico e a taxa de mortalidade, apresentando-se inversamente proporcional com a idade dos pacientes.

\title{
A B S T R A C T
}

\begin{abstract}
Objectives: There are few publications which relate the injury severity score (ISS) to the thoracolumbar burst fractures. For that reason and for the frequency in which they occur, we have evaluated the severity of the trauma in these patients. Methods: We have evaluated 190 burst fractures in the spinal cord according to Denis, using the codes of Abbreviated Injury Scales (AIS) for the calculation of the ISS, which uses the three parts of the human body with major severity. These lesions are a squared number and the results are summed up. Results: Among 190 cases evaluated, the median value of the ISS was 13 and the average was 14,4. Males presented a higher ISS than females. The young adult patients presented an average and a median value of the ISS higher than the old patients. The higher the ISS is, the longer the hospitalization period is, except for the patients with the ISS over 35. The fractures in thoracic level show the ISS higher than the rest. The ISS is directly related to surgical treatment and mortality. Conclusion: The ISS values which were found show that a less severe trauma can cause a burst thoracic or lumbar spinal cord fracture. The value of the ISS has not shown correlation to the sex and the fracture level, but it is proportional to the hospitalization period, the surgical treatment and the mortality rate. This result shows a value which is inversely proportional to the age of the patients.
\end{abstract}

Key words: Lumbar vertebrae. Spine thoracic vertebrae. Spinal injuries. Multiple trauma. 


\section{REFERENCIAS}

1. Denis F. The three column spine and its significance in the classification of acute thoracolumbar spinal injuries. Spine. 1983; 8(8):817-31.

2. Champion HR, Copes WS, Sacco WJ, Lawnick MM, Keast SL, Bain LW Jr, Flanagan ME, Frey CF. The Major Trauma Outcome Study: establishing national norms for trauma care. J Trauma. 1990; 30(11):1356-65.

3. Akmal M, Trivedi R, Sutcliffe J. Functional outcome in trauma patients with spinal injury. Spine. 2003; 28(2):180-5.

4. Committe on Medical Aspects Automotive Safety: Rating the Severity of Tissue Damage- 1. The Abbreviated Injury Scale. JAMA. 1971; 215: 277- 280.

5. Baker SP, O'Neill B, Haddon W Jr, Long WB. The injury severity score: a method for describing patients with multiple injuries and evaluating emergency care. J Trauma. 1974; 14(3):187-96.

6. Hansen ST Jr. The type-IIIC tibial fracture. Salvage or amputation. J Bone Joint Surg Am. 1987; 69(6):799-800.

7. Meek RN, Vivoda EE, Pirani S. Comparison of mortality of patients with multiple injuries according to type of fracture treatment-a retrospective age- and injury-matched series. Injury. 1986; 17(1):2-4.

8. Gennari TD, Koizumi MS. Determinação do nível de gravidade do trauma. Rev Saúde Pública. 1995; 29(5)333-41.

9. Long WB, Bachulis BL, Hynes GD. Accuracy and relationship of mechanisms of injury, trauma score, and injury severity score in identifying major trauma. Am J Surg. 1986; 151(5):581-4.

10. Lindsey RW, Dick W, Nunchuck S, Zach G. Residual intersegmental spinal mobility following limited pedicle fixation of thoracolumbar spine fractures with the fixateur interne. Spine. 1993; 18(4):474-8.

11. Rimoldi RL, Zigler JE, Capen DA, Hu SS. The effect of surgical intervention on rehabilitation time in patients with thoracolumbar and lumbar spinal cord injuries. Spine. 1992; 17(12):1443-9.

12. Aebi M, Etter C, Kehl T, Thalgott J. Stabilization of the lower thoracic and lumbar spine with the internal spinal skeletal fixation system. Indications, techniques, and first results of treatment. Spine. 1987; 12(6):544-51.

13. Schwab CW, Young G, Civil I, Ross SE, Talucci R, Rosenberg L, Shaikh K, O'Malley K, Camishion RC. DRG reimbursement for trauma: the demise of the trauma center (the use of ISS grouping as an early predictor of total hospital cost). J Trauma. 1988; 28(7):93946.
14. Meves R, Avanzi O. Correlation between neurological deficit and spinal canal compromise in 198 patients with thoracolumbar and lumbar fractures. Spine. 2005; 30(7):787-91.

15. Long WB, Bachulis BL, Hynes GD. Accuracy and relationship of mechanisms of injury, trauma score, and injury severity score in identifying major trauma. Am J Surg. 1986; 151(5):581-4.

16. Hill $A B$, Fleiszer DM, Brown RA. Chest trauma in a Canadian urban setting--implications for trauma research in Canada. J Trauma. 1991; 31(7):971-3.

17. Dai LY, Yao WF, Cui YM, Zhou Q. Thoracolumbar fractures in patients with multiple injuries: diagnosis and treatment-a review of 147 cases. J Trauma. 2004; 56(2):348-55.

18. Sheridan R, Peralta R, Rhea J, Ptak T, Novelline R. Reformatted visceral protocol helical computed tomographic scanning allows conventional radiographs of the thoracic and lumbar spine to be eliminated in the evaluation of blunt trauma patients. J Trauma. 2003; 55(4):665-9.

19. Willén J, Lindahl S, Nordwall A. Unstable thoracolumbar fractures. A comparative clinical study of conservative treatment and Harrington instrumentation. Spine. 1985; 10(2):111-22.

20. Frankel HL, Rozycki GS, Ochsner MG, Harviel JD, Champion HR. Indications for obtaining surveillance thoracic and lumbar spine radiographs. J Trauma. 1994; 37(4):673-6.

21. Fontijne WP, de Klerk LW, Braakman R, Stijnen T, Tanghe HL, Steenbeek $R$, van Linge $B$. CT scan prediction of neurological deficit in thoracolumbar burst fractures. J Bone Joint Surg Br. 1992: 74(5):683-5

Recebido em 01/09/2008

Aceito para publicação em 31/10/2008

Conflito de interesse: nenhum

Fonte de financiamento: nenhuma

\section{Como citar este artigo:}

Rezende R, Avanzi O. Importância do Injury Severity Score (ISS) no manejo das fraturas toracolombares do tipo explosão. Rev Col Bras Cir. [periódico na Internet] 2009; 36(1). Disponível em URL: http:// www.scielo.br/rcbc

\section{Endereço para correspondência:}

Osmar Avanzi

coluna@santacasasp.org.br 